\title{
Comparative Cost Analysis of Endoscopic versus Microscopic Endonasal Transsphenoidal Surgery for Pituitary Adenomas
}

\author{
Chikezie Ikechukwu Eseonu $^{1}$ Karim ReFaey ${ }^{1,2}$ Oscar Garcia ${ }^{1}$ Roberto Salvatori ${ }^{3}$ \\ Alfredo Quinones-Hinojosa ${ }^{1,2}$
}

${ }^{1}$ Department of Neurosurgery, Johns Hopkins University, Baltimore, Maryland, United States

2 Department of Neurological Surgery, Mayo Clinic, Jacksonville, Florida, United States

${ }^{3}$ Department of Endocrinology, Diabetes, and Metabolism, Johns Hopkins University, Baltimore, Maryland, United States

\begin{abstract}
Address for correspondence Alfredo Quinones-Hinojosa, MD, Brain Tumor Stem Cell Laboratory, Department of Neurological Surgery, Mayo Clinic, Florida, 4500 San Pablo Road S, Jacksonville, FL 32224 (e-mail: Quinones-Hinojosa.Alfredo@mayo.edu).
\end{abstract}

J Neurol Surg B 2018;79:131-138.

\begin{abstract}
Keywords

- adenoma

- costs

- endoscopic

- microscopic

- pituitary

- transsphenoidal

Objective This study presents a comparative analysis of cost efficacy between the microscopic and endoscopic transsphenoidal approaches, evaluating neurological outcome, extent of resection (EOR), and inpatient hospital costs.

Design This study was a retrospective chart review.

Setting This study was conducted at a tertiary care center.

Participants The study group consisted of 68 patients with transsphenoidal surgeries between January 2007 and January 2014.

Main Outcome Measures Two-sample t-tests and Pearson's chi-square test evaluated inpatient costs, quality-adjusted life years (QALYs), volumetric EOR, and neurological outcomes.

Results Total inpatient costs per patient was $\$ 22,853$ in the microscopic group and less $(\$ 19,736)$ in the endoscopic group $(p=0.049)$. Operating room costs were $\$ 5,974$ in the microscopic group and lower in the endoscopic group $(\$ 5,045$; $p=0.038)$. Operative time was 203.6 minutes in the microscopic group and 166.3 minutes in the endoscopic group $(p=0.032)$. The QALY score, length of hospital stay, and postoperative outcomes were found to be similar between the two cohorts. Multivariate linear regression modeling suggested that length of stay $(p<0.001)$ and operative time ( $p=0.008)$ were important factors that influenced total inpatient costs following transsphenoidal surgery.

Conclusion This study shows that transsphenoidal surgery is more cost effective with the endoscopic approach than with the microscopic approach and depends on efficiency in the operating room as well as reduction in the length of hospitalization.
\end{abstract}

\section{Introduction}

There are nearly 5,000 surgeries for pituitary adenomas in the United States each year, and the cost of these procedures can total up to $\$ 100$ million annually. ${ }^{1-3}$ The transsphenoidal surgical approach presents a common technique to resect pituitary adenomas. The two methods employed for the transsphenoidal approach include the use of the operating microscope, the microscopic approach, which can be endonasal, transseptal, or sublabial, or the use of an endoscope, received

February 6, 2017

accepted after revision

June 17, 2017

published online

August 8, 2017 (c) 2018 Georg Thieme Verlag KG Stuttgart · New York
DOI https://doi.org/ 10.1055/s-0037-1604484. ISSN 2193-6331. 
the endoscopic approach, which is endonasal. ${ }^{3}$ Both the microscopic and endoscopic approaches are used throughout the world, and which technique provides better extent of tumor resection continues to be debated.

Less information is available in the literature about the cost effectiveness associated with these two techniques. The United States healthcare system is adding increased emphasis on the treatment efficacy when determining physician and hospital payment. ${ }^{4,5}$ Physician reimbursement now relies on a patient's hospital experience, efficient clinical process, and outcome. This makes understanding the surgical efficacy and hospital costs essential for transsphenoidal surgery. ${ }^{6}$ The literature has yet to present both a cost and surgical outcome comparative analysis for the microscopic and endoscopic transsphenoidal approaches.

In this study, we present a single-surgeon experience and the first comparative analysis of both the surgical efficacy and inpatient costs between the microscopic and endoscopic endonasal transsphenoidal approaches, evaluating neurological outcome, extent of resection (EOR), and inpatient costs. We present a unique cost analysis between the endoscopic and microscopic transsphenoidal approaches that also evaluates the effect of EOR and patient outcomes on the variability of inpatient costs.

\section{Methods}

\section{Patient Selection}

Patients were selected based on the following criteria: (1) age $\geq 18$ years, (2) had a pituitary adenoma based on pathology, (3) an elective procedure, (4) no major comorbidities that would require an elongated hospitalization, and (5) had endonasal surgery via the transsphenoidal corridor using the microscopic approach, the microscopic group. From the criteria, 34 patients were found to have undergone a microscopic approach transsphenoidal surgery for a pituitary adenoma between January 2007 and January 2014. To reduce the confounding factors and increase the study efficiency, a matched-pairs design was performed for preoperative tumor size, tumor pathology, preoperative Karnofsky performance status (KPS), and preoperative visual deficits. The matched group consisted of a retrospective cohort of 34 patients who underwent an endoscopic endonasal transsphenoidal surgery between the same time period and met the inclusion criteria mentioned above. Only patients who underwent transsphenoidal surgery while the primary surgeon was conducting both microscopic and endoscopic approaches were included in this study. A single surgeon at a single institution performed all the surgeries. An endocrinologist was involved with all preoperative evaluation for both nonfunctional and functional adenomas.

\section{Surgical Procedure}

Microscopic approach: A microscopic mononostril approach with a nasal speculum was used by the primary surgeon. The tumor was removed using suction, ring curettes, and pituitary rongeurs. Following the tumor resection, the sellar space was reconstructed with an autologous abdominal fat graft, hemostatic cellulose polymer (SURGICEL; Ethicon, Somerville, New Jersey, United States), and dural sealant (DuraSeal, Medtronics; Minneapolis, Minnesota, United States). ${ }^{7-9}$

Endoscopic approach: An endoscopic technique using a mononostril approach was conducted by the primary surgeon. The middle turbinate was lateralized and a mucosal nasoseptal flap was created when needed. The tumor resection was conducted with the surgical assistant holding the endoscope and the surgeon conducting the tumor resection with suction, angled curettes, and pituitary rongeurs. The sellar reconstruction was performed as mentioned in the microscopic approach. ${ }^{7-9}$ There was no need of an otolaryngologist for the exposure in the endoscopic procedure.

\section{Postoperative Management}

Following surgery, patients were monitored and managed for diabetes insipidus and followed up postoperatively at 1 month, 6 months, and then annually.

Progression free survival (PFS) was determined based on the time of the initial surgery to when there was tumor recurrence (regrowth of the tumor) or when tumor progression occurred (increased growth of a residual tumor) based on postoperative magnetic resonance imaging (MRI) reported by a neuroradiologist who was blinded to the groups. For functional tumors, recurrence also included the situation with an evidence of hormonal oversecretion with follow-up laboratories. The quality adjusted life year (QALY) score quantified the health gain from the surgical approaches accounting for life expectancy and quality of life. We used the equation QALY $=$ PFS $\times$ (postoperative KPS at 6 months/ 100) as previously used in the literature. ${ }^{10,11}$

\section{Tumor Volume Analysis}

Preoperative tumor volume was determined based on the preoperative MRI, performed 1 day before surgery, and postoperative tumor volume was based on the postoperative MRI taken 48 hours after surgery. A volumetric analysis on the T1-weighted coronal sequence MRI with and without contrast was used to determine the volumes. The software, OsiriX (Pixmeo), was used to calculate the tumor area of each coronal cut $(1.5-3.0 \mathrm{~mm})$, and the sum of these values determined the volume, as we have previously described, by a clinician who was blinded to the cohorts. ${ }^{11-14}$ The EOR was calculated based on the formula (preoperative volume - postoperative volume)/preoperative volume.

\section{Cost Analysis}

The total inpatient costs during the hospitalization included costs for room/board, operating room (including operative equipment and surgeon fee), staff, medications, laboratory, inpatient supplies, inpatient physical/occupational therapy, diagnostic imaging, and anesthesia charge time. These data were obtained from the hospital finance department that contained the cost of treatment for each patient. Costs were based on the day of surgery until hospital discharge and did not include preoperative days that may have been spent inpatient. The QALY was the generic measurement to assess 
disease burden, where one QALY equated to 1 year in perfect health. The cost per QALY was determined by dividing the total inpatient costs by the QALY score. The incremental costeffectiveness ratio (ICER) is the ratio of the change in the mean total inpatient cost between the two techniques to the change in the QALYs of the two techniques, ICER $=$ (cost endo - cost micro)/(QALY endo - QALY micro), which shows the additional cost that is associated with the added benefit of one intervention over the other. ${ }^{15,16}$

\section{Statistical Analysis}

Univariate statistics were performed to generate descriptive statistics, which were reported as the number of subjects and percentage for categorical variables, mean, and standard deviations for continuous parametric variables. Parametric comparisons between the two groups were performed using the two-sample $t$-test, and nonparametric comparisons were performed using rank-sum test. Categorical comparisons between the two groups (microscopic versus endoscopic) were performed using Pearson's chi-square test. The significance of differences was evaluated according to a type I error rate threshold of $\alpha=0.05$. A biostatistician contributed to the statistical analysis of this study (O.G.). All statistical analyses were performed using STATA 14 (StataCorp, College Station, Texas, United States).
This study was approved by the appropriate Institutional Review Board (IRB), and the requirement for written informed consent was waived by the IRB.

\section{Results}

\section{Preoperative Characteristics}

Sixty-eight patients met the inclusion criteria for this study and were treated by a single neurosurgeon for a pituitary adenoma with an endonasal transsphenoidal surgery at a single institution. Thirty-four patients underwent a transsphenoidal surgery with the operating microscope and were case-controlled matched to thirty-four patients who underwent an endoscopic transsphenoidal surgery. Mean age, gender, and preoperative symptoms were found to be similar in the two cohorts ( - Table 1 ).

\section{Tumor Characteristics}

There were $29.4 \%$ functional and $70.6 \%$ nonfunctional adenomas in the microscopic group and $23.5 \%$ functional and $76.5 \%$ nonfunctional adenomas in the endoscopic group $(p=0.582)$. Each prolactinoma patient underwent surgery after medical treatment failure with cabergoline, and all acromegaly patients had medical treatment failure with octreotide. Preoperative tumor volume was 5.14 and $6.03 \mathrm{~cm}^{3}$ in the

Table 1 Preoperative characteristics of 68 patients

\begin{tabular}{|l|l|l|l|}
\hline & \multicolumn{2}{|l|}{ Type of Surgery } & \\
\hline Characteristic & $\begin{array}{l}\text { Microscopic } \\
(n=34)\end{array}$ & $\begin{array}{l}\text { Endoscopic } \\
(n=34)\end{array}$ & $p$-Value \\
\hline Age, mean (SD) & $45.6(16.4)$ & $50.7(1.93)$ & 0.138 \\
\hline Male gender, $n$ (\%) & $20(58.8)$ & $18(52.9)$ & 0.625 \\
\hline Race & & & 0.775 \\
\hline White, $n$ (\%) & $23(67.6)$ & $19(55.9)$ & \\
\hline Black, $n$ (\%) & $7(20.6)$ & $9(26.5)$ & \\
\hline Hispanic, $n$ (\%) & $3(8.8)$ & $4(11.8)$ & \\
\hline Asian, $n$ (\%) & $1(2.9)$ & $2(5.9)$ & \\
\hline KPS, mean (SD) & $85.0(7.88)$ & $87.4(8.98)$ & 0.255 \\
\hline Seizure, $n$ (\%) & $2(5.8)$ & $0(0.0)$ & 0.493 \\
\hline Headache, $n$ (\%) & $20(58.8)$ & $15(44.1)$ & 0.225 \\
\hline Nausea/Vomiting, $n$ (\%) & $3(8.8)$ & $4(11.8)$ & 0.690 \\
\hline Diplopia, $n$ (\%) & $4(11.8)$ & $4(11.8)$ & 1.000 \\
\hline Proptosis, $n$ (\%) & $1(2.9)$ & $1(2.9)$ & 1.000 \\
\hline Visual acuity deficit, $n(\%)$ & $15(44.1)$ & $18(52.9)$ & 0.467 \\
\hline Visual field defect, $n$ (\%) & $8(23.5)$ & $8(23.5)$ & 1.000 \\
\hline Confusion, $n$ (\%) & $2(5.9)$ & $2(5.9)$ & 1.000 \\
\hline CN3 deficit, $n$ (\%) & $3(8.8)$ & $1(2.9)$ & 0.614 \\
\hline CN4 deficit, $n$ (\%) & $1(2.9)$ & $1(2.9)$ & 1.000 \\
\hline CN5 deficit, $n$ (\%) & $0(0.00)$ & $0(0.00)$ & - \\
\hline CN6 deficit, $n$ (\%) & $0(0.00)$ & $0(0.00)$ & - \\
\hline
\end{tabular}

Abbreviations: CN, cranial nerve; KPS, Karnofsky performance status; SD, standard deviation. 
134 Pituitary Adenomas: Cost Analysis Eseonu et al.

Table 2 Pituitary tumor characteristics of 68 patients

\begin{tabular}{|l|l|l|l|}
\hline & \multicolumn{2}{|l|}{ Type of surgery } & \\
\hline Characteristic & $\begin{array}{l}\text { Microscopic } \\
(n=34)\end{array}$ & $\begin{array}{l}\text { Endoscopic } \\
(n=34)\end{array}$ & p-Value \\
\hline Tumor type, $n(\%)$ & & & 0.582 \\
\hline Functional, $n$ (\%) & $10(29.4)$ & $8(23.5)$ & \\
\hline Nonfunctional, $n$ (\%) & $24(70.6)$ & $26(76.5)$ & \\
\hline Macroadenoma & $23(67.6)$ & $24(70.6)$ & 0.793 \\
\hline Microadenoma & $11(32.6)$ & $10(29.4)$ & \\
\hline Secreting tumors & & & 0.682 \\
\hline Prolactinoma, $n$ (\%) & $1(2.9)$ & $1(2.9)$ & \\
\hline ACTH Prod, $n$ (\%) & $6(17.6)$ & $5(14.7)$ & \\
\hline Growth hormone, $n(\%)$ & $2(5.9)$ & $3(8.8)$ & \\
\hline TSH Prod, $n$ (\%) & $0(0.0)$ & $1(2.9)$ & \\
\hline FSH/LH, $n$ (\%) & $1(2.9)$ & $0(0.0)$ & 0.549 \\
\hline Knosp grade & & & \\
\hline $0-2$ & $26(76.6)$ & $28(82.4)$ & \\
\hline $3-4$ & $8(23.5)$ & $6(17.6)$ & 0.882 \\
\hline EOR, mean (SD) & $84.8 \%(20.2)$ & $85.5 \%(16.6)$ & 0.207 \\
\hline PreOp tumor volume, $\mathrm{cm}^{3}$ mean (SD) & $5.14(5.22)$ & $6.03(3.71)$ & 0.296 \\
\hline PostOp tumor volume, $\mathrm{cm}^{3}$ mean (SD) & $1.21(3.54)$ & $1.18(1.79)$ & 0.032 \\
\hline Operative times (min), mean (SD) & $203.6(61.2)$ & $166.3(44.9)$ & 0.115 \\
\hline LOS, mean, d (range) & $3.38(1-8)$ & $2.53(1-6)$ & \\
\hline
\end{tabular}

Abbreviations: ACTH, adrenocorticotropic hormone; EOR, extent of resection; $\mathrm{FSH}$, follicle stimulating hormone; $\mathrm{GH}$, growth hormone; $\mathrm{LH}$, luteinizing hormone; LOS, length of stay; PostOp, postoperative; PreOp, preoperative; SD, standard deviation; TSH, thyroid stimulating hormone.

microscopic and endoscopic groups, respectively ( $p=0.207)$. The mean EOR for the microscopic and endoscopic groups was 84.8 and $85.5 \%$, respectively. Complete $100 \%$ resections were not seen $(0 \%)$ in the Knosp 3-4 group for the microscopic cohort, but was observed in one (16.7\%) of the Knosp 3-4 group for the endoscopic cohort. Operative time was 203.6 minutes in the microscopic group and 166.3 minutes in the endoscopic group ( $p=0.032$, - Table 2 ).

\section{Postoperative Characteristics}

Postoperative cerebral spinal fluid (CSF) leak was observed in two (5.9\%) microscopic patients and one (2.9\%) endoscopic patient. All three patients were treated with a lumbar drain. Additional postoperative outcomes and complications were found to occur in similar frequencies between the two cohorts ( - Table 3). At 1-month follow-up clinic visit, adrenal insufficiency was observed in $11.8 \%$ of the microscopic and $14.7 \%$ of the endoscopic patients $(p=0.720)$. All the patients who experienced adrenal insufficiency (four microscopic patients and five endoscopic patients) had preoperative Cushing's disease. Hypothyroidism was found in $2.9 \%$ of the microscopic patients and none of the endoscopic patients $(p=0.314)$. Transient diabetes insipidus (DI) was found in $20.6 \%$ of the microscopic patients and $14.7 \%$ of the endoscopic patients $(p=0.525)$. DI patients from both cohorts were initially treated with subcutaneous vasopressin that
Table 3 Postoperative outcomes and complications of 68 patients

\begin{tabular}{|l|l|l|l|}
\hline & \multicolumn{2}{|l|}{ Type of surgery } & \\
\hline Characteristic & $\begin{array}{l}\text { Microscopic } \\
(n=34)\end{array}$ & $\begin{array}{l}\text { Endoscopic } \\
(n=34)\end{array}$ & $p$-Value \\
\hline Postoperative KPS & $84.7(7.48)$ & $87.1(8.71)$ & 0.237 \\
\hline Vision change & $12(35.3)$ & $17(50.0)$ & 0.440 \\
\hline $\begin{array}{l}\text { Vision improved, } \\
n(\%)\end{array}$ & $20(58.8)$ & $16(47.1)$ & \\
\hline $\begin{array}{l}\text { Vision maintained, } \\
n(\%)\end{array}$ & $2(5.9)$ & $1(2.9)$ & \\
\hline $\begin{array}{l}\text { Vision worsened, } \\
n(\%)\end{array}$ & $2(5.9)$ & $1(2.9)$ & 0.555 \\
\hline CSF leak, $n(\%)$ & $0(0.00)$ & $1(2.9)$ & 0.314 \\
\hline ICA violated, $n(\%)$ & $0(0.00)$ & $1(2.9)$ & 0.314 \\
\hline Stroke, $n(\%)$ & $0(0.00)$ & $0(0.00)$ & - \\
\hline Meningitis, $n(\%)$ & $0(0.00)$ & $0(0.00)$ & - \\
\hline Epistaxis, $n(\%)$ & $0(0.00)$ & $0(0.00)$ & - \\
\hline Sinusitis, $n(\%)$ & $0(0.00)$ & $0(0.00)$ & - \\
\hline Mucocele, $n(\%)$ & & \\
\hline
\end{tabular}

Abbreviations: CSF, cerebrospinal fluid; ICA, internal carotid artery; KPS, Karnofsky performance status. 
Table 4 Average hospital cost by category for 68 patients

\begin{tabular}{|l|l|l|l|}
\hline \multicolumn{2}{|l|}{ Type of surgery } & \\
\hline Category, mean \$ (range) & $\begin{array}{l}\text { Microscopic } \\
(n=34)\end{array}$ & $\begin{array}{l}\text { Endoscopic } \\
(n=34)\end{array}$ & $p$-Value \\
\hline Total inpatient charge & $22,853(12,508-43,511)$ & $19,736(10,515-32,659)$ & 0.049 \\
\hline Room and board & $6,455(2,417-16,383)$ & $5,213(1,596-11,023)$ & 0.198 \\
\hline Operating room & $5,974(2,8825-10,941)$ & $5,045(2,240-9,888)$ & 0.038 \\
\hline Pharmacy & $383(76-1380)$ & $374(48-1,109)$ & 0.686 \\
\hline Radiology & $3,474(1,000-6,086)$ & $3,303(1,789-5,304)$ & 0.548 \\
\hline Laboratory & $1,656(988-3,210)$ & $1,396(695-2,386)$ & 0.084 \\
\hline Medical supplies & $3,855(400-9,144)$ & $3,959(1,432-7,641)$ & 0.552 \\
\hline Inpatient P/O therapy & $490(0-1,901)$ & $451(0-1,732)$ & 0.555 \\
\hline Other ${ }^{\text {a }}$ & $385(0-1,663)$ & $211(0-1,359)$ & 0.081 \\
\hline PFS, mean y (SD) & $4.86(2.4)$ & $4.92(1.9)$ & 0.917 \\
\hline QALY, mean (SD) & $4.07(1.9)$ & $4.25(1.6)$ & 0.679 \\
\hline Cost per QALY & $9,636(2,382-55,504)$ & $5,450(2,846-42,122)$ & 0.145 \\
\hline
\end{tabular}

Abbreviations: P/O, physical/occupational; PFS, progression free survival; QALY, quality adjusted life year; SD, standard deviation.

${ }^{a}$ Other costs include intraoperative monitoring time, electroencephalogram (EEG) \& electrocardiogram (EKG) charge time, and blood transfusions.

was transitioned to an oral desmopressin dose that was tapered off in the outpatient setting by an endocrinologist.

\section{Cost Analysis}

The total cost for the inpatient stay was $\$ 22,853$ and $\$ 19,736$ for the microscopic and endoscopic groups, respectively $(p=0.049)$. Operating room costs were $\$ 5,974$ and $\$ 5,045$ for the microscopic and endoscopic groups, respectively $(p=0.038)$. Additional hospital costs did not differ significantly by category (-Table 4). The mean quality adjusted life year (QALY) score was 4.07 in the microscopic group and 4.92 in the endoscopic group $(p=0.679)$. The mean ICER showed that the mean incremental cost per QALY for the endoscopic patients was $\$ 17,266$ less than that for the microscopic patients. In other words, the ICER presents a theoretical value, which indicates that there is a mean saving of $\$ 17,266$ for each QALY gained when a patient with a pituitary adenoma undergoes an endoscopic transsphenoidal approach instead of a microscopic transsphenoidal approach.

\section{Predictors of Cost}

A linear regression model was created to determine the primary drivers of total inpatient costs for transsphenoidal surgery. An exploratory univariate analysis was conducted to find covariates of interest that had significant associations with the total inpatient cost ( $\mathbf{- T a b l e ~} \mathbf{5}$ ). A multivariate model was then designed using the identified variables, namely, length of stay (LOS), operative time, and functional adenoma. The LOS ( $<<0.001,-$ Fig. 1) and operative time ( $p=0.015$, - Fig. 2 ) were found to have significant associations with total inpatient cost, where significant increases in LOS or operative time were likely to produce significant changes in total inpatient cost.
Table 5 Univariate analysis of variables affecting total inpatient costs for transsphenoidal surgery. The three significant variables were found to be LOS, operating room costs, and functional adenomas

\begin{tabular}{|l|l|l|}
\hline Variable & $\mathrm{R}^{2}$ & $p$-Value \\
\hline LOS & 0.471 & $<0.001$ \\
\hline Operative time & 0.087 & 0.015 \\
\hline Functional adenoma & 0.073 & 0.025 \\
\hline Postoperative complication ${ }^{\mathrm{a}}$ & 0.009 & 0.447 \\
\hline Preoperative tumor volume & 0.048 & 0.174 \\
\hline EOR & $<0.001$ & 0.901 \\
\hline Large tumor $\left(>8.9 \mathrm{~cm}^{3}\right)$ & 0.038 & 0.110 \\
\hline Age & 0.010 & 0.429 \\
\hline Gender & 0.024 & 0.211 \\
\hline
\end{tabular}

Abbreviation: EOR, extent of resection; LOS, length of stay.

ancludes vision loss, new visual defects, diabetes insipidus, syndrome of inappropriate antidiuretic hormone, cerebral spinal fluid leak, internal carotid injury, stroke, meningitis, epistaxis, sinusitis, or mucocele.

\section{Discussion}

The two most common surgical transsphenoidal approaches for a pituitary adenoma include the microscopic and endoscopic techniques. Over the past decade, many surgeons have transitioned from the microscopic approach to the endoscopic approach, but little is known about the cost effectiveness of either technique. ${ }^{17-21}$ We present a single-surgeon, single-institution, cost analysis comparing microscopic and 


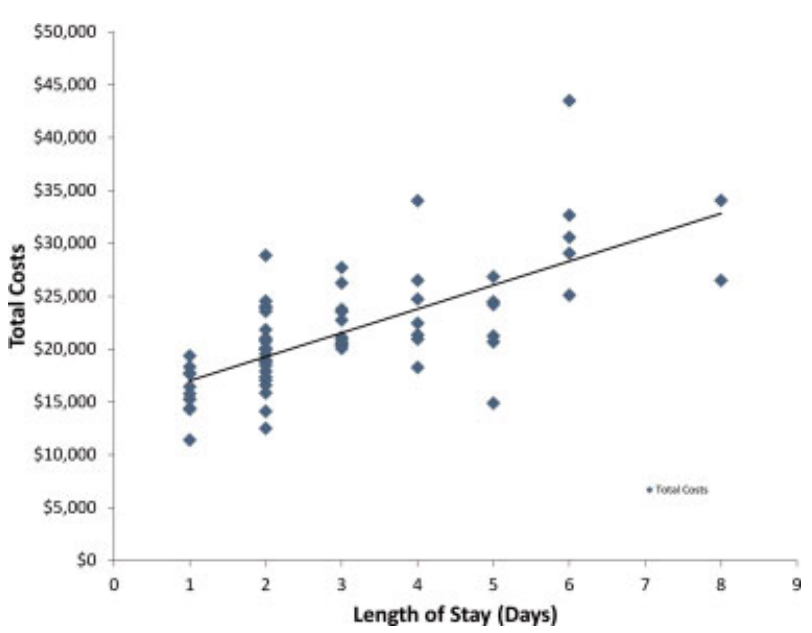

Fig. 1 Linear regression demonstrating a significant association between length of hospital stay and total inpatient cost $(p<0.001)$.

endoscopic transsphenoidal surgery for pituitary adenomas and evaluate the impact of postoperative outcomes and EOR on costs. Previous studies have looked at the cost comparisons of these two groups, but there has been no assessment of the postoperative outcomes from these cohorts that can play an integral part with inpatient hospital costs. ${ }^{22,23}$ To our knowledge, we present the first cost and outcome analysis for the transsphenoidal microscopic and endoscopic approaches. We found that total inpatient costs, operative costs, and operative times for patients who underwent an endoscopic transsphenoidal approach were less than those who underwent a microscopic transsphenoidal pituitary surgery, and that LOS and operative time were important predictors of total inpatient costs.

\section{Factors Influencing Inpatient Costs}

A few previous studies have tried to evaluate costs of endoscopic and microscopic pituitary surgery. Rudmik et $\mathrm{al}^{3}$ evaluated endoscopic and microscopic costs by reviewing a series of meta-analyses and using a Markov decision tree model that simulated the treatment and costs of a patient

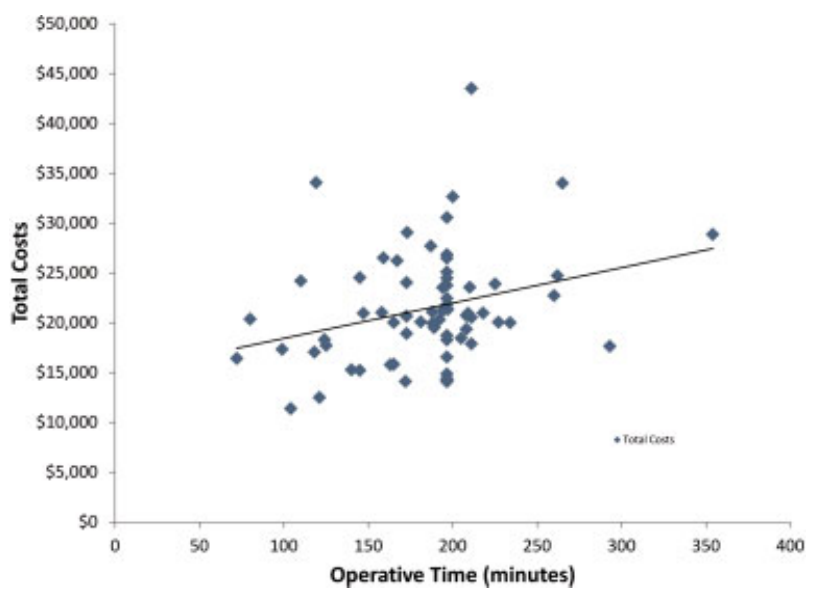

Fig. 2 Linear regression demonstrating a significant association between operative time and total inpatient cost $(p=0.015)$. undergoing a pituitary adenoma resection. This study presented a theoretical cost analysis that did not look at a specific patient population or outcomes. Little et $\mathrm{al}^{22}$ analyzed the endoscopic technique of one surgeon to the microscopic technique of another surgeon. However, different postoperative management strategies between the two surgeons added additional variables that could have affected postoperative costs. Our study was based on a patient population treated at a single institution by a single neurosurgeon who directed the postoperative care for both groups, which provided a consistent protocol for postoperative management. We further evaluated patient outcome to identify any significant variables that could skew the total cost results.

Inpatient costs for microscopic transsphenoidal surgeries have been reported in the literature to range from $\$ 23,757$ to $\$ 74,703$, while the endoscopic endonasal costs range from $\$ 12,513$ to $\$ 72,311 .^{3,22,23}$ Our study found that our inpatient costs averaged $\$ 22,853$ and $\$ 19,736$ for the microscopic and endoscopic approaches, respectively. The cost utility showed that the mean cost per QALY was larger in the microscopic group $(\$ 9,636)$ than the endoscopic group $(\$ 5,450)$. The ICER showed mean savings of $\$ 17,266$ per QALY gained when a patient underwent an endoscopic transsphenoidal instead of the microscopic approach, suggesting that the added cost to gain an extra QALY (one year of perfect health) by using the microscopic approach instead of the endoscopic approach would be $\$ 17,266$. Although there was no significant difference in the QALY between the two cohorts, the endoscopic group had significantly lower inpatient total costs with a general trend of higher QALY scores, which was reflected in the calculation of the ICER.

Previous studies have used QALY scores to evaluate pituitary tumor patients; however, these utilized theoretical QALY scores that did not assess the condition of actual patients. ${ }^{3,24}$ Our study used PFS and KPS to assess the QALY score as has previously been described. ${ }^{10,11}$ Although these pituitary adenomas are benign lesions, given that visual deficits from pituitary tumors can greatly impact the KPS of a patient, these provide a good measurement for quality of life. In addition, pituitary adenomas have a tendency to recur or show growth from a residual tumor following surgery, which is well documented with serial imaging. As a result, utilizing PFS provides a good indicator for tumor recurrence/regrowth, which affects the QALY score and allows the QALY for our study to provide a good estimation for tumor burden and quality of life. To ensure adequate comparisons of the QALY score, the cohorts were case matched for preoperative KPS to adjust for any preoperative conditions that could impact the postoperative care and LOS for the patients.

Not all factors that affect surgical costs are well understood, and higher surgical fees do not necessarily produce better quality outcomes. ${ }^{25}$ Oosmanally et $\mathrm{al}^{23}$ determined that the LOS was a key factor influencing costs for transsphenoidal surgery and also showed that compared with the sublabial approach, the endonasal endoscopic approach required less postoperative pain medication that may aid 
in reducing the pharmacy costs. Other studies have also looked at $\operatorname{LOS}^{2,22}$ as a significant driver of hospital costs following pituitary tumor surgery. We found that LOS and operative time were both key components of total inpatient costs. The LOS was found to account for a significant amount of the total cost, with the cost of room and board making up to $29 \%$ of the microscopic patient's total cost and $25 \%$ of the endoscopic patient's total cost. Operating room costs comprised of 24 and $27 \%$ of the total inpatient cost for the microscopic and endoscopic patients, respectively.

\section{Reducing Costs}

Based on our study, certain factors that may aid in decreasing inpatient costs following transsphenoidal surgery included efforts to reduce operative time and provide efficient postoperative inpatient care, since these components accounted for a large percentage of total cost. At our institution, we have transitioned to the endoscopic approach given that over time we found that our endoscopic operative time became shorter while not compromising the EOR or outcomes. For this cohort, we utilized a mononostril approach that lateralized the middle turbinate to allow for quick access to the sellar region and helped reduce operative time. ${ }^{26}$ Ultimately, a surgeon's comfort with a given technique and the surgical team's experience are likely to help decrease operative times for transsphenoidal surgery.

In addition to reducing operative time, shortening the length of a patient's hospital stay can also help reduce total inpatient costs. The difficulty with trying to reduce LOS is that patient care can be compromised if an adequate duration of postoperative care is not achieved. We found that by involving our endocrinology team with both the preoperative assessment and the inpatient care of the patient, the endocrine team could quickly become familiar with the patient's postoperative endocrine issues, which provided for an easier transition to outpatient endocrine management. Studies have shown that patients with Cushing's disease can have higher inpatient and outpatient hospital costs. $^{22,27}$ The two cohorts in our study were matchedpaired for preoperative conditions, such as tumor size, pathology, preoperative KPS, and preoperative visual deficits to compare groups with similar amounts of functional adenomas and preoperative conditions to prevent bias in our cost calculations. Our univariate regression analysis showed that functional adenomas could also play a role in total inpatient cost, likely due to the increased cost for serial laboratories and the increased LOS that maybe required to follow postoperative hormonal levels for a hormonal cure. Involving an endocrinologist can allow for more efficient management of hormonal conditions and help minimize these additional costs.

\section{Patient Selection}

For our patient cohort, we reviewed patients who were operated on during a time period when the senior neurosurgeon was performing both the microscopic and endoscopic surgical approaches to maintain similar hospital costs and account for inflation. For patient selection for either the microscopic or endoscopic approach, the patient's preoperative characteristics and presentation did not affect which surgical approach was offered. Preoperative tumor size and Knosp grades were similar between our two cohorts; however, during the early stage of transitioning to the endoscopic approach tumors that appeared on imaging to involve the carotid artery were selected to undergo a microscopic approach due to the theoretically higher risk of intraoperative bleeding. Although this study evaluated tumor volumetrics and extent of lateral tumor invasion, additional factors such as the contour of the tumor or extension over the planum are variables that were not assessed in our volumetric studies and that could also bias surgical approach selection. Ultimately, surgical approach selection comes down to the comfort and experience of the surgeon and the surgical team.

\section{Limits of the Study}

This study is a retrospective analysis that contains the biases inherent in all nonrandomized controlled studies. Although our single-surgeon perspective allows for consistency with perioperative management between the patient cohorts, institutional differences with charges and fees for surgical service may show a wide variation in inpatient costs for different practices. Since only a single-surgeon did both the endoscopic and microscopic approaches, this study could not evaluate additional operative costs that might have resulted from having an otolaryngologist cosurgeon with the endoscopic approach. In our practice, we reserved the use of endoscopic assistance with otolaryngology for giant cancerous tumors of patients who had multiple surgeries or radiation treatment for cancer in the past and required extensive exposure and reconstruction. In addition, we did not evaluate long-term outpatient costs that could differ if an otolaryngologist, who could perform routine outpatient nasal scope exams, was involved and routinely followed a transsphenoidal surgery patient for potential nasal complications.

\section{Conclusion}

The treatment of pituitary adenomas can present a substantial cost for the inpatient stay. In our experience with a single surgeon doing both procedures, the endoscopic transsphenoidal approach provided less inpatient total costs and shorter operative times than the microscopic approach, although both approaches achieved similar operative outcomes. We found that operative time and LOS were predictors of total inpatient costs, suggesting that optimizing these factors may help reduce overall inpatient costs.

\section{References}

1 Barker FG II, Klibanski A, Swearingen B. Transsphenoidal surgery for pituitary tumors in the United States, 1996-2000: mortality, morbidity, and the effects of hospital and surgeon volume. J Clin Endocrinol Metab 2003;88(10):4709-4719

2 Lee JY, Bohman LE, Bergsneider M. Contemporary neurosurgical techniques for pituitary tumor resection. J Neurooncol 2014; 117(03):437-444 
3 Rudmik L, Starreveld YP, Vandergrift WA, Banglawala SM, Soler ZM. Cost-effectiveness of the endoscopic versus microscopic approach for pituitary adenoma resection. Laryngoscope 2015; 125(01):16-24

4 Chien AT, Rosenthal MB. Medicare's physician value-based payment modifier-will the tectonic shift create waves? N Engl J Med 2013;369(22):2076-2078

5 Shoemaker W. The cost of quality: how VBP scores correlate with hospital costs. Healthc Financ Manage 2012;66(10):50-56

6 McLaughlin N, Khalessi AA, Martin NA. Health care economics in neurosurgery: there is no turning back. Neurosurg Focus 2014;37 (05):E1

7 Sanai N, Quiñones-Hinojosa A, Narvid J, Kunwar S. Safety and efficacy of the direct endonasal transsphenoidal approach for challenging sellar tumors. J Neurooncol 2008;87(03):317-325

8 Frazier JL, Chaichana K, Jallo GI, Quiñones-Hinojosa A. Combined endoscopic and microscopic management of pediatric pituitary region tumors through one nostril: technical note with case illustrations. Childs Nerv Syst 2008;24(12):1469-1478

9 Eseonu CI, ReFaey K, Rincon-Torroella J, et al. Endoscopic versus microscopic transsphenoidal approach for pituitary adenomas: comparison of outcomes during the transition of methods of a single surgeon. World Neurosurg 2017;97:317-325

10 Martino J, Gomez E, Bilbao JL, Dueñas JC, Vázquez-Barquero A. Cost-utility of maximal safe resection of WHO grade II gliomas within eloquent areas. Acta Neurochir (Wien) 2013;155(01): 41-50

11 Eseonu CI, Rincon-Torroella J, ReFaey K, Quinones-Hinojosa A. The cost of brain surgery: awake versus asleep craniotomy for perirolandic region tumors. Neurosurgery 2017. Doi: 10.1093/neuros/nyx022

12 Chaichana KL, Jusue-Torres I, Navarro-Ramirez R, et al. Establishing percent resection and residual volume thresholds affecting survival and recurrence for patients with newly diagnosed intracranial glioblastoma. Neuro Oncol 2014;16(01):113-122

13 Chaichana KL, Jusue-Torres I, Lemos AM, et al. The butterfly effect on glioblastoma: is volumetric extent of resection more effective than biopsy for these tumors? J Neurooncol 2014;120(03): 625-634

14 Eseonu CI, Rincon-Torroella J, ReFaey K, et al. Awake craniotomy versus craniotomy under general anesthesia for perirolandic gliomas: evaluating perioperative complications and extent of resection. Neurosurgery 2016. Doi: 10.1093/neuros/nyx023
15 Kim S, Mortaz Hedjri S, Coyte PC, Rampersaud YR. Cost-utility of lumbar decompression with or without fusion for patients with symptomatic degenerative lumbar spondylolisthesis. Spine J 2012;12(01):44-54

16 Parker SL, Adogwa O, Davis BJ, et al. Cost-utility analysis of minimally invasive versus open multilevel hemilaminectomy for lumbar stenosis. J Spinal Disord Tech 2013;26(01):42-47

17 Doglietto F, Prevedello DM, Jane JA Jr, Han J, Laws ER Jr. Brief history of endoscopic transsphenoidal surgery-from philipp bozzini to the first world congress of endoscopic skull base surgery. Neurosurg Focus 2005;19(06):E3

18 Jho HD, Carrau RL. Endoscopic endonasal transsphenoidal surgery: experience with 50 patients. J Neurosurg 1997;87(01):44-51

19 Jho HD, Carrau RL, Ko Y, Daly MA. Endoscopic pituitary surgery: an early experience. Surg Neurol 1997;47(03):213-222; discussion 222-223

20 Liu JK, Das K, Weiss MH, Laws ER Jr, Couldwell WT. The history and evolution of transsphenoidal surgery. J Neurosurg 2001;95(06): 1083-1096

21 Mehta GU, Lonser RR, Oldfield EH. The history of pituitary surgery for Cushing disease. J Neurosurg 2012;116(02):261-268

22 Little AS, Chapple K, Jahnke H, White WL. Comparative inpatient resource utilization for patients undergoing endoscopic or microscopic transsphenoidal surgery for pituitary lesions. J Neurosurg 2014;121(01):84-90

23 Oosmanally N, Paul JE, Zanation AM, Ewend MG, Senior BA, Ebert CS Jr. Comparative analysis of cost of endoscopic endonasal minimally invasive and sublabial-transseptal approaches to the pituitary. Int Forum Allergy Rhinol 2011;1(04):242-249

24 Ament J, Yang Z, Tertulien P, Shahlaie K. Comparing the costeffectiveness of endoscopic and microscopic approaches to transphenoidal pituitary surgery. J Neurol Surg B Skull Base 2014. Doi: $10.1055 / \mathrm{s}-0034-1370450$

25 Newhouse JP, Garber AM. Geographic variation in health care spending in the United States: insights from an Institute of Medicine report. JAMA 2013;310(12):1227-1228

26 Eseonu CI, ReFaey K, Pamias-Portalatin E, et al. Three-hand endoscopic endonasal transsphenoidal surgery: experience with an anatomy-preserving mononostril approach technique. Oper Neurosurg 2017 DOI: https://doi.org/10.1093/ons/opx110

27 Swearingen B, Wu N, Chen SY, Pulgar S, Biller BM. Health care resource use and costs among patients with cushing disease. Endocr Pract 2011;17(05):681-690 